Acta Crystallographica Section E

Structure Reports

Online

ISSN 1600-5368

\section{3-Ethynyl-2,2,5,5-tetramethyl-1-oxyl-3- pyrroline}

\section{Olga Frolow, Jan W. Bats* and Joachim W. Engels}

Institut für Organische Chemie, Universität Frankfurt, Max-von-Laue-Strasse 7, D-60438 Frankfurt am Main, Germany

Correspondence e-mail: bats@chemie.uni-frankfurt.de

Received 7 July 2009; accepted 8 July 2009

Key indicators: single-crystal X-ray study; $T=167 \mathrm{~K}$; mean $\sigma(\mathrm{C}-\mathrm{C})=0.002 \AA$; $R$ factor $=0.062 ; w R$ factor $=0.157 ;$ data-to-parameter ratio $=27.3$.

The five-membered ring of the title compound, $\mathrm{C}_{10} \mathrm{H}_{14} \mathrm{NO}$, is almost planar [mean deviation from best plane $=0.006$ (1) $\AA$ ]. The $\mathrm{N}-\mathrm{O}$ bond is in the plane of the five-membered ring. The molecule is positioned about a pseudo-mirror plane at $y=$ 0.375. In the crystal, molecules are connected by intermolecular $\mathrm{C}-\mathrm{H}$... O contacts into layers parallel to (010).

\section{Related literature}

For the preparation of the title compound, see: Schiemann et al. (2007). For its application as a spin label, see: Schiemann et al. (2007); Piton et al. (2007). For the crystal structure of a related compound, see: Fritscher et al. (2002).

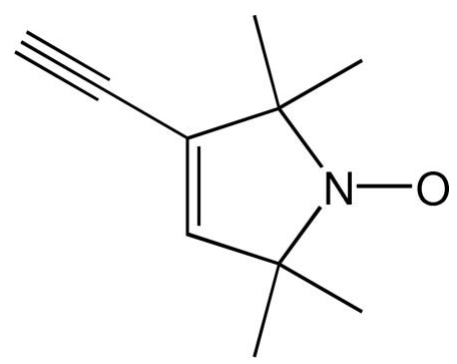

\section{Experimental}

Crystal data

$\mathrm{C}_{10} \mathrm{H}_{14} \mathrm{NO}$

$M_{r}=164.22$
Monoclinic, $P 2_{1} / c$

$a=7.9326(15) \AA$

$b=19.058$ (4) $\AA$

$c=6.5989(11) \AA$

$\beta=104.333(14)^{\circ}$

$V=966.6(3) \AA^{3}$

Data collection

Siemens SMART 1K CCD diffractometer

Absorption correction: multi-scan (SADABS; Sheldrick, 2000)

$T_{\min }=0.870, T_{\max }=0.995$

Refinement

$R\left[F^{2}>2 \sigma\left(F^{2}\right)\right]=0.062$

$w R\left(F^{2}\right)=0.157$

$S=1.19$

3301 reflections

121 parameters

$Z=4$

Mo $K \alpha$ radiation

$\mu=0.07 \mathrm{~mm}^{-1}$

$T=167 \mathrm{~K}$

$0.60 \times 0.55 \times 0.07 \mathrm{~mm}$

16848 measured reflections 3301 independent reflections 2214 reflections with $I>2 \sigma(I)$

$R_{\text {int }}=0.035$

Table 1

Hydrogen-bond geometry $\left(\AA{ }^{\circ}\right)$.

\begin{tabular}{lllll}
\hline$D-\mathrm{H} \cdots A$ & $D-\mathrm{H}$ & $\mathrm{H} \cdots A$ & $D \cdots A$ & $D-\mathrm{H} \cdots A$ \\
\hline $\mathrm{C} 2-\mathrm{H} 2 A \cdots \mathrm{O} 1^{\mathrm{i}}$ & $0.975(19)$ & $2.441(18)$ & $3.3907(18)$ & $164.6(14)$ \\
$\mathrm{C} 6-\mathrm{H} 6 A \cdots \mathrm{O} 1^{\mathrm{ii}}$ & $0.98(2)$ & $2.20(2)$ & $3.174(2)$ & $171.2(17)$
\end{tabular}

Symmetry codes: (i) $x, y, z-1$; (ii) $x-1, y, z-1$.

Data collection: SMART (Siemens, 1995); cell refinement: SMART; data reduction: SAINT (Siemens, 1995); program(s) used to solve structure: SHELXS97 (Sheldrick, 2008); program(s) used to refine structure: SHELXL97 (Sheldrick, 2008); molecular graphics: SHELXTL (Sheldrick, 2008); software used to prepare material for publication: SHELXL97.

Supplementary data and figures for this paper are available from the IUCr electronic archives (Reference: NC2152).

\section{References}

Fritscher, J., Beyer, M. \& Schiemann, O. (2002). Chem. Phys. Lett. 364, 393 401.

Piton, N., Mu, Y., Stock, G., Prisner, T. F., Schiemann, O. \& Engels, J. W. (2007). Nucleic Acids Res. 35, 3128-3143.

Schiemann, O., Piton, N., Plackmeyer, J., Bode, B. E., Prisner, T. F. \& Engels, J. W. (2007). Nat. Protoc. 2, 904-923.

Sheldrick, G. M. (2000). SADABS. University of Göttingen, Germany.

Sheldrick, G. M. (2008). Acta Cryst. A64, 112-122.

Siemens (1995). SMART and SAINT. Siemens Analytical X-ray Instruments Inc., Madison, Wisconsin, USA. 


\section{supporting information}

Acta Cryst. (2009). E65, o1848 [doi:10.1107/S1600536809026725]

\section{3-Ethynyl-2,2,5,5-tetramethyl-1-oxyl-3-pyrroline}

\section{Olga Frolow, Jan W. Bats and Joachim W. Engels}

\section{S1. Comment}

For EPR measurements of RNA, DNA or proteins, the occurrence of paramagnetic species is required. The title compound is a nitroxide spin label compound. Its synthesis has been reported by Schiemann et al. (2007). The application for DNA and RNA labeling has been described by Schiemann et al. (2007) and Piton et al. (2007). Here we report the crystal structure of the compound.

The molecular structure of the title compound is shown in Fig. 1. The five-membered ring is almost planar: the mean deviation from the best plane is 0.006 (1) $\AA$. The molecule approximately has mirror symmetry and is positioned about a pseudo-mirror plane at $y=0.375$. The $\mathrm{N}$ atom is planar and deviates by only 0.006 (2) $\AA$ from the plane through $\mathrm{C} 1, \mathrm{C} 4$ and O1. A related molecule with a very similar conformation of the 3-ethynyl-2,2,5,5-tetramethyl-1-oxyl-3-pyrroline group has been reported by Fritscher et al. (2002).

The molecules are connected by intermolecular $\mathrm{C}-\mathrm{H} \cdots \mathrm{O}$ contacts to layers parallel to $\left[\begin{array}{lll}0 & 1 & 0\end{array}\right]$ (Fig. 2 and Table 1).

\section{S2. Experimental}

The preparation of the title compound has been reported by Schiemann et al. (2007). Crystals were obtained by recrystallization from ethanol.

\section{S3. Refinement}

The $\mathrm{H}$ atoms at $\mathrm{C} 2$ and $\mathrm{C} 6$ were taken from a difference Fourier synthesis and were refined with isotropic thermal parameters. The remaining $\mathrm{H}$ atoms were geometrically positioned using: $\mathrm{C}_{\text {methyl }}-\mathrm{H}=0.98 \AA$ and $U_{\text {iso }}(H)=1.5 U_{\text {eq }}\left(\mathrm{C}_{\text {methyl }}\right)$. The torsion angles about the $C-\mathrm{C}_{\text {methyl }}$ bonds were refined for the methyl groups 


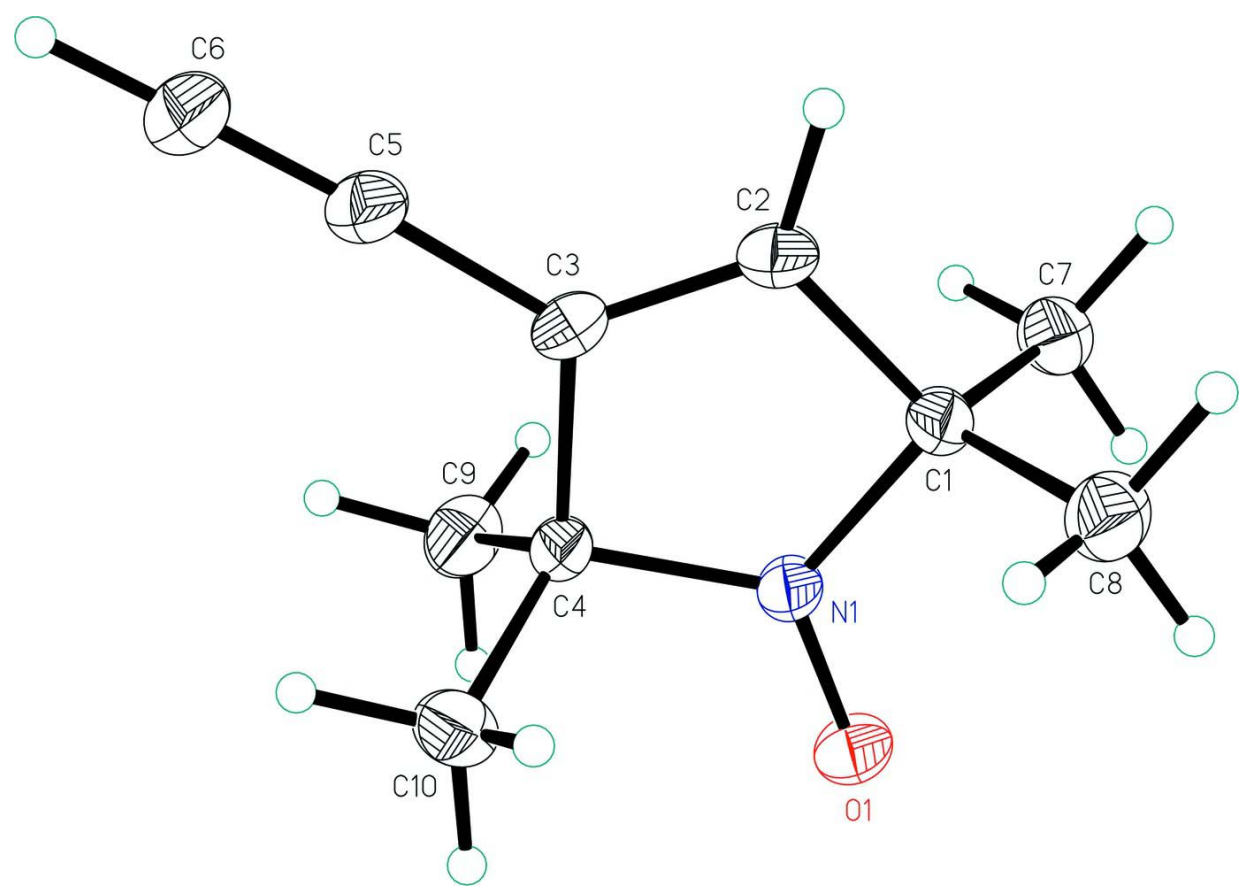

\section{Figure 1}

The structure of the title compound shown with $50 \%$ probability displacement ellipsoids. The $\mathrm{H}$ atoms are drawn as small spheres of arbitrary radius.

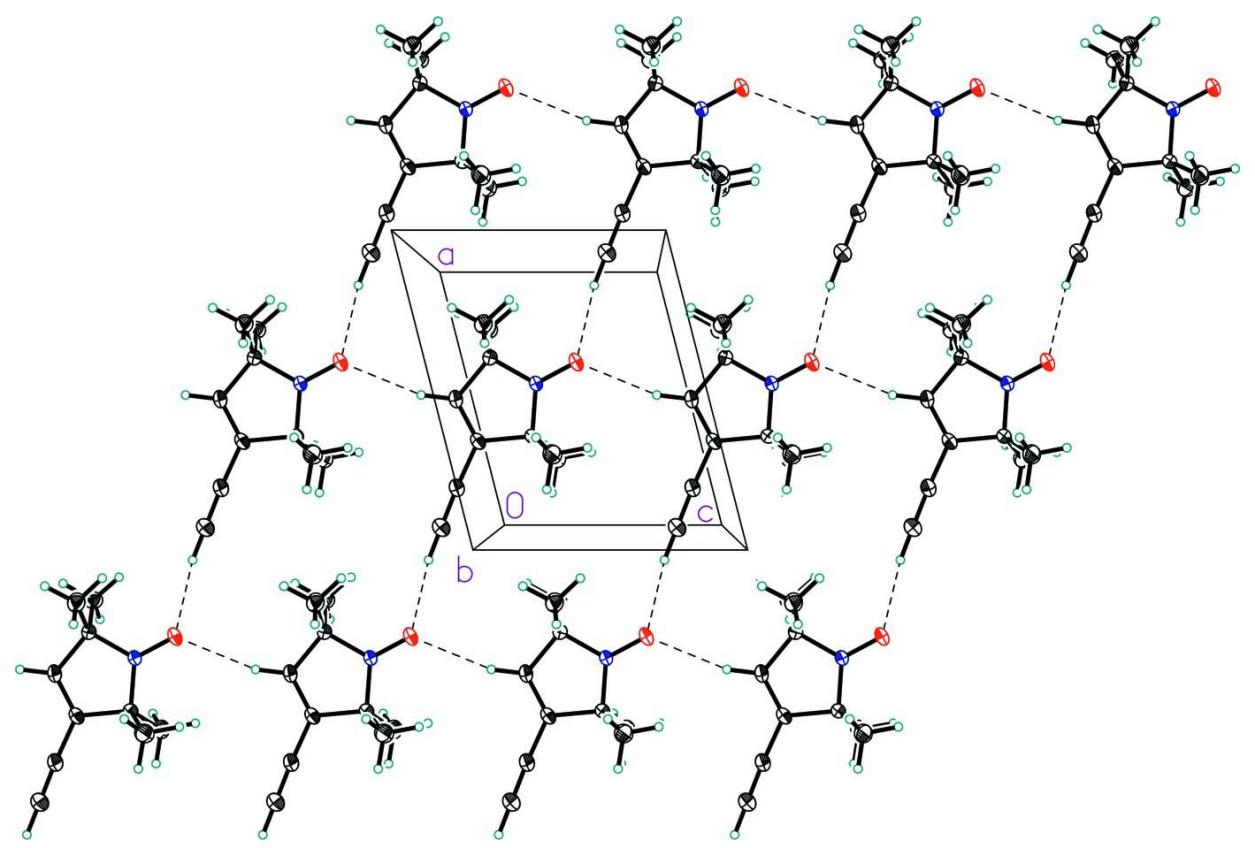

Figure 2

A hydrogen bonded layer of molecules, viewed down the $b$ axis. Intermolecular $\mathrm{C}-\mathrm{H} \cdots \mathrm{O}$ contacts are shown as dashed lines. 


\section{3-Ethynyl-2,2,5,5-tetramethyl-1-oxyl-3-pyrroline}

Crystal data

$\mathrm{C}_{10} \mathrm{H}_{14} \mathrm{NO}$

$M_{r}=164.22$

Monoclinic, $P 2_{1} / c$

Hall symbol: -P 2ybc

$a=7.9326(15) \AA$

$b=19.058(4) \AA$

$c=6.5989(11) \AA$

$\beta=104.333(14)^{\circ}$

$V=966.6(3) \AA^{3}$

$Z=4$

\section{Data collection}

Siemens SMART 1K CCD diffractometer

Radiation source: normal-focus sealed tube Graphite monochromator

$\omega$ scans

Absorption correction: multi-scan

(SADABS; Sheldrick, 2000)

$T_{\min }=0.870, T_{\max }=0.995$

Refinement

Refinement on $F^{2}$

Least-squares matrix: full

$R\left[F^{2}>2 \sigma\left(F^{2}\right)\right]=0.062$

$w R\left(F^{2}\right)=0.157$

$S=1.19$

3301 reflections

121 parameters

0 restraints

Primary atom site location: structure-invariant direct methods
$F(000)=356$

$D_{\mathrm{x}}=1.129 \mathrm{Mg} \mathrm{m}^{-3}$

Mo $K \alpha$ radiation, $\lambda=0.71073 \AA$

Cell parameters from 130 reflections

$\theta=3-23^{\circ}$

$\mu=0.07 \mathrm{~mm}^{-1}$

$T=167 \mathrm{~K}$

Plate, yellow

$0.6 \times 0.55 \times 0.07 \mathrm{~mm}$

16848 measured reflections

3301 independent reflections

2214 reflections with $I>2 \sigma(I)$

$R_{\text {int }}=0.035$

$\theta_{\max }=32.4^{\circ}, \theta_{\min }=2.1^{\circ}$

$h=-11 \rightarrow 11$

$k=-27 \rightarrow 28$

$l=-9 \rightarrow 9$

Secondary atom site location: difference Fourier map

Hydrogen site location: inferred from neighbouring sites

$\mathrm{H}$ atoms treated by a mixture of independent and constrained refinement

$w=1 /\left[\sigma^{2}\left(F_{\mathrm{o}}^{2}\right)+(0.05 P)^{2}+0.35 P\right]$ where $P=\left(F_{\mathrm{o}}^{2}+2 F_{\mathrm{c}}^{2}\right) / 3$

$(\Delta / \sigma)_{\max }=0.005$

$\Delta \rho_{\max }=0.44$ e $\AA^{-3}$

$\Delta \rho_{\min }=-0.23$ e $\AA^{-3}$

\section{Special details}

Geometry. All e.s.d.'s (except the e.s.d. in the dihedral angle between two 1.s. planes) are estimated using the full covariance matrix. The cell e.s.d.'s are taken into account individually in the estimation of e.s.d.'s in distances, angles and torsion angles; correlations between e.s.d.'s in cell parameters are only used when they are defined by crystal symmetry. An approximate (isotropic) treatment of cell e.s.d.'s is used for estimating e.s.d.'s involving 1.s. planes.

Refinement. Refinement of $F^{2}$ against ALL reflections. The weighted $R$-factor $w R$ and goodness of fit $S$ are based on $F^{2}$, conventional $R$-factors $R$ are based on $F$, with $F$ set to zero for negative $F^{2}$. The threshold expression of $F^{2}>\sigma\left(F^{2}\right)$ is used only for calculating $R$-factors (gt) etc. and is not relevant to the choice of reflections for refinement. $R$-factors based on $F^{2}$ are statistically about twice as large as those based on $F$, and $R$ - factors based on ALL data will be even larger.

Fractional atomic coordinates and isotropic or equivalent isotropic displacement parameters $\left(\AA^{2}\right)$

\begin{tabular}{lllll}
\hline & $x$ & $y$ & $z$ & $U_{\text {iso }} * / U_{\text {eq }}$ \\
\hline O1 & $0.62303(13)$ & $0.37389(6)$ & $0.53230(15)$ & $0.0287(3)$ \\
$\mathrm{N} 1$ & $0.54492(14)$ & $0.37427(7)$ & $0.33858(17)$ & $0.0219(2)$ \\
C1 & $0.63968(16)$ & $0.37208(8)$ & $0.1714(2)$ & $0.0208(3)$
\end{tabular}




$\begin{array}{lllll}\text { C2 } & 0.48802(18) & 0.37338(8) & -0.0187(2) & 0.0230(3) \\ \text { C3 } & 0.33449(17) & 0.37599(8) & 0.0304(2) & 0.0213(3) \\ \text { C4 } & 0.35322(16) & 0.37802(8) & 0.2659(2) & 0.0203(3) \\ \text { C5 } & 0.16502(18) & 0.37655(8) & -0.1101(2) & 0.0257(3) \\ \text { C6 } & 0.0205(2) & 0.37657(10) & -0.2191(2) & 0.0326(3) \\ \text { C7 } & 0.7461(2) & 0.30471(8) & 0.1866(3) & 0.0286(3) \\ \text { H7A } & 0.6683 & 0.2641 & 0.1719 & 0.043^{*} \\ \text { H7B } & 0.8307 & 0.3028 & 0.3228 & 0.043^{*} \\ \text { H7C } & 0.8077 & 0.3040 & 0.0750 & 0.043^{*} \\ \text { C8 } & 0.7560(2) & 0.43669(9) & 0.1864(3) & 0.0298(3) \\ \text { H8A } & 0.6849 & 0.4792 & 0.1763 & 0.045^{*} \\ \text { H8B } & 0.8146 & 0.4360 & 0.0719 & 0.045^{*} \\ \text { H8C } & 0.8433 & 0.4363 & 0.3206 & 0.045^{*} \\ \text { C9 } & 0.2700(2) & 0.31455(9) & 0.3439(2) & 0.0302(4) \\ \text { H9A } & 0.3192 & 0.2714 & 0.3017 & 0.045^{*} \\ \text { H9B } & 0.1441 & 0.3153 & 0.2833 & 0.045^{*} \\ \text { H9C } & 0.2933 & 0.3162 & 0.4967 & 0.045^{*} \\ \text { C10 } & 0.2884(2) & 0.44689(9) & 0.3368(3) & 0.0315(4) \\ \text { H10A } & 0.3487 & 0.4862 & 0.2898 & 0.047^{*} \\ \text { H10B } & 0.3119 & 0.4475 & 0.4897 & 0.047^{*} \\ \text { H10C } & 0.1629 & 0.4512 & 0.2764 & 0.047^{*} \\ \text { H2A } & 0.503(2) & 0.3735(9) & -0.161(3) & 0.029(4)^{*} \\ \text { H6A } & -0.098(3) & 0.3781(10) & -0.308(3) & 0.045(6)^{*} \\ \end{array}$

Atomic displacement parameters $\left(\AA^{2}\right)$

\begin{tabular}{lllllll}
\hline & $U^{11}$ & $U^{22}$ & $U^{33}$ & $U^{12}$ & $U^{13}$ & $U^{23}$ \\
\hline O1 & $0.0254(5)$ & $0.0447(6)$ & $0.0133(4)$ & $0.0007(5)$ & $-0.0005(3)$ & $-0.0003(5)$ \\
N1 & $0.0177(5)$ & $0.0338(6)$ & $0.0135(5)$ & $0.0002(5)$ & $0.0025(4)$ & $0.0002(5)$ \\
C1 & $0.0185(5)$ & $0.0273(7)$ & $0.0171(5)$ & $-0.0002(5)$ & $0.0050(4)$ & $0.0001(6)$ \\
C2 & $0.0253(6)$ & $0.0288(7)$ & $0.0142(5)$ & $-0.0006(6)$ & $0.0039(4)$ & $-0.0005(5)$ \\
C3 & $0.0216(6)$ & $0.0239(6)$ & $0.0159(5)$ & $-0.0007(5)$ & $-0.0001(4)$ & $0.0000(5)$ \\
C4 & $0.0162(5)$ & $0.0274(7)$ & $0.0165(5)$ & $0.0000(5)$ & $0.0027(4)$ & $-0.0013(5)$ \\
C5 & $0.0252(6)$ & $0.0334(8)$ & $0.0176(6)$ & $-0.0015(6)$ & $0.0034(5)$ & $-0.0006(6)$ \\
C6 & $0.0262(7)$ & $0.0466(10)$ & $0.0229(7)$ & $-0.0002(7)$ & $0.0019(5)$ & $-0.0017(7)$ \\
C7 & $0.0248(7)$ & $0.0312(8)$ & $0.0298(8)$ & $0.0050(6)$ & $0.0065(6)$ & $-0.0030(6)$ \\
C8 & $0.0288(7)$ & $0.0351(9)$ & $0.0260(8)$ & $-0.0081(6)$ & $0.0081(6)$ & $0.0007(6)$ \\
C9 & $0.0278(7)$ & $0.0388(9)$ & $0.0241(8)$ & $-0.0073(6)$ & $0.0065(6)$ & $0.0054(6)$ \\
C10 & $0.0292(8)$ & $0.0379(9)$ & $0.0266(8)$ & $0.0085(7)$ & $0.0053(6)$ & $-0.0071(7)$ \\
& & & & & & \\
\hline
\end{tabular}

Geometric parameters $\left(\AA,{ }^{\circ}\right)$

\begin{tabular}{llll}
\hline $\mathrm{O} 1-\mathrm{N} 1$ & $1.2752(14)$ & $\mathrm{C} 6-\mathrm{H} 6 \mathrm{~A}$ & $0.97(2)$ \\
$\mathrm{N} 1-\mathrm{C} 4$ & $1.4787(16)$ & $\mathrm{C} 7-\mathrm{H} 7 \mathrm{~A}$ & 0.9800 \\
$\mathrm{~N} 1-\mathrm{C} 1$ & $1.4815(17)$ & $\mathrm{C} 7-\mathrm{H} 7 \mathrm{~B}$ & 0.9800 \\
$\mathrm{C} 1-\mathrm{C} 2$ & $1.5079(18)$ & $\mathrm{C} 7-\mathrm{H} 7 \mathrm{C}$ & 0.9800 \\
$\mathrm{C} 1-\mathrm{C} 7$ & $1.526(2)$ & $\mathrm{C} 8-\mathrm{H} 8 \mathrm{~A}$ & 0.9800 \\
$\mathrm{C} 1-\mathrm{C} 8$ & $1.527(2)$ & $\mathrm{C} 8-\mathrm{H} 8 \mathrm{~B}$ & 0.9800
\end{tabular}




\begin{tabular}{|c|c|c|c|}
\hline $\mathrm{C} 2-\mathrm{C} 3$ & $1.336(2)$ & $\mathrm{C} 8-\mathrm{H} 8 \mathrm{C}$ & 0.9800 \\
\hline $\mathrm{C} 2-\mathrm{H} 2 \mathrm{~A}$ & $0.975(19)$ & C9-H9A & 0.9800 \\
\hline $\mathrm{C} 3-\mathrm{C} 5$ & $1.4317(18)$ & $\mathrm{C} 9-\mathrm{H} 9 \mathrm{~B}$ & 0.9800 \\
\hline $\mathrm{C} 3-\mathrm{C} 4$ & $1.5248(18)$ & $\mathrm{C} 9-\mathrm{H} 9 \mathrm{C}$ & 0.9800 \\
\hline $\mathrm{C} 4-\mathrm{C} 10$ & $1.525(2)$ & $\mathrm{C} 10-\mathrm{H} 10 \mathrm{~A}$ & 0.9800 \\
\hline $\mathrm{C} 4-\mathrm{C} 9$ & $1.527(2)$ & $\mathrm{C} 10-\mathrm{H} 10 \mathrm{~B}$ & 0.9800 \\
\hline $\mathrm{C} 5-\mathrm{C} 6$ & $1.193(2)$ & $\mathrm{C} 10-\mathrm{H} 10 \mathrm{C}$ & 0.9800 \\
\hline $\mathrm{O} 1-\mathrm{N} 1-\mathrm{C} 4$ & $122.07(11)$ & $\mathrm{C} 1-\mathrm{C} 7-\mathrm{H} 7 \mathrm{~B}$ & 109.5 \\
\hline $\mathrm{O} 1-\mathrm{N} 1-\mathrm{C} 1$ & $122.43(11)$ & $\mathrm{H} 7 \mathrm{~A}-\mathrm{C} 7-\mathrm{H} 7 \mathrm{~B}$ & 109.5 \\
\hline $\mathrm{C} 4-\mathrm{N} 1-\mathrm{C} 1$ & $115.50(10)$ & $\mathrm{C} 1-\mathrm{C} 7-\mathrm{H} 7 \mathrm{C}$ & 109.5 \\
\hline $\mathrm{N} 1-\mathrm{C} 1-\mathrm{C} 2$ & $99.88(10)$ & $\mathrm{H} 7 \mathrm{~A}-\mathrm{C} 7-\mathrm{H} 7 \mathrm{C}$ & 109.5 \\
\hline $\mathrm{N} 1-\mathrm{C} 1-\mathrm{C} 7$ & $110.43(12)$ & $\mathrm{H} 7 \mathrm{~B}-\mathrm{C} 7-\mathrm{H} 7 \mathrm{C}$ & 109.5 \\
\hline $\mathrm{C} 2-\mathrm{C} 1-\mathrm{C} 7$ & $112.54(12)$ & $\mathrm{C} 1-\mathrm{C} 8-\mathrm{H} 8 \mathrm{~A}$ & 109.5 \\
\hline $\mathrm{N} 1-\mathrm{C} 1-\mathrm{C} 8$ & $109.81(12)$ & $\mathrm{C} 1-\mathrm{C} 8-\mathrm{H} 8 \mathrm{~B}$ & 109.5 \\
\hline $\mathrm{C} 2-\mathrm{C} 1-\mathrm{C} 8$ & $112.67(12)$ & $\mathrm{H} 8 \mathrm{~A}-\mathrm{C} 8-\mathrm{H} 8 \mathrm{~B}$ & 109.5 \\
\hline $\mathrm{C} 7-\mathrm{C} 1-\mathrm{C} 8$ & $111.00(12)$ & $\mathrm{C} 1-\mathrm{C} 8-\mathrm{H} 8 \mathrm{C}$ & 109.5 \\
\hline $\mathrm{C} 3-\mathrm{C} 2-\mathrm{C} 1$ & $112.74(12)$ & $\mathrm{H} 8 \mathrm{~A}-\mathrm{C} 8-\mathrm{H} 8 \mathrm{C}$ & 109.5 \\
\hline $\mathrm{C} 3-\mathrm{C} 2-\mathrm{H} 2 \mathrm{~A}$ & $124.7(11)$ & $\mathrm{H} 8 \mathrm{~B}-\mathrm{C} 8-\mathrm{H} 8 \mathrm{C}$ & 109.5 \\
\hline $\mathrm{C} 1-\mathrm{C} 2-\mathrm{H} 2 \mathrm{~A}$ & $122.5(11)$ & $\mathrm{C} 4-\mathrm{C} 9-\mathrm{H} 9 \mathrm{~A}$ & 109.5 \\
\hline $\mathrm{C} 2-\mathrm{C} 3-\mathrm{C} 5$ & $127.55(13)$ & $\mathrm{C} 4-\mathrm{C} 9-\mathrm{H} 9 \mathrm{~B}$ & 109.5 \\
\hline $\mathrm{C} 2-\mathrm{C} 3-\mathrm{C} 4$ & $112.53(11)$ & $\mathrm{H} 9 \mathrm{~A}-\mathrm{C} 9-\mathrm{H} 9 \mathrm{~B}$ & 109.5 \\
\hline $\mathrm{C} 5-\mathrm{C} 3-\mathrm{C} 4$ & $119.92(12)$ & $\mathrm{C} 4-\mathrm{C} 9-\mathrm{H} 9 \mathrm{C}$ & 109.5 \\
\hline $\mathrm{N} 1-\mathrm{C} 4-\mathrm{C} 3$ & $99.34(10)$ & $\mathrm{H} 9 \mathrm{~A}-\mathrm{C} 9-\mathrm{H} 9 \mathrm{C}$ & 109.5 \\
\hline $\mathrm{N} 1-\mathrm{C} 4-\mathrm{C} 10$ & $109.88(12)$ & $\mathrm{H} 9 \mathrm{~B}-\mathrm{C} 9-\mathrm{H} 9 \mathrm{C}$ & 109.5 \\
\hline $\mathrm{C} 3-\mathrm{C} 4-\mathrm{C} 10$ & $112.29(12)$ & $\mathrm{C} 4-\mathrm{C} 10-\mathrm{H} 10 \mathrm{~A}$ & 109.5 \\
\hline $\mathrm{N} 1-\mathrm{C} 4-\mathrm{C} 9$ & $110.37(12)$ & $\mathrm{C} 4-\mathrm{C} 10-\mathrm{H} 10 \mathrm{~B}$ & 109.5 \\
\hline $\mathrm{C} 3-\mathrm{C} 4-\mathrm{C} 9$ & $112.47(12)$ & $\mathrm{H} 10 \mathrm{~A}-\mathrm{C} 10-\mathrm{H} 10 \mathrm{~B}$ & 109.5 \\
\hline $\mathrm{C} 10-\mathrm{C} 4-\mathrm{C} 9$ & $111.82(13)$ & $\mathrm{C} 4-\mathrm{C} 10-\mathrm{H} 10 \mathrm{C}$ & 109.5 \\
\hline $\mathrm{C} 6-\mathrm{C} 5-\mathrm{C} 3$ & $176.86(16)$ & $\mathrm{H} 10 \mathrm{~A}-\mathrm{C} 10-\mathrm{H} 10 \mathrm{C}$ & 109.5 \\
\hline $\mathrm{C} 5-\mathrm{C} 6-\mathrm{H} 6 \mathrm{~A}$ & $178.2(12)$ & $\mathrm{H} 10 \mathrm{~B}-\mathrm{C} 10-\mathrm{H} 10 \mathrm{C}$ & 109.5 \\
\hline $\mathrm{C} 1-\mathrm{C} 7-\mathrm{H} 7 \mathrm{~A}$ & 109.5 & & \\
\hline $\mathrm{O} 1-\mathrm{N} 1-\mathrm{C} 1-\mathrm{C} 2$ & $179.84(13)$ & $\mathrm{C} 1-\mathrm{N} 1-\mathrm{C} 4-\mathrm{C} 3$ & $1.46(17)$ \\
\hline $\mathrm{C} 4-\mathrm{N} 1-\mathrm{C} 1-\mathrm{C} 2$ & $-1.06(17)$ & $\mathrm{O} 1-\mathrm{N} 1-\mathrm{C} 4-\mathrm{C} 10$ & $62.65(18)$ \\
\hline $\mathrm{O} 1-\mathrm{N} 1-\mathrm{C} 1-\mathrm{C} 7$ & $61.15(17)$ & $\mathrm{C} 1-\mathrm{N} 1-\mathrm{C} 4-\mathrm{C} 10$ & $-116.46(14)$ \\
\hline $\mathrm{C} 4-\mathrm{N} 1-\mathrm{C} 1-\mathrm{C} 7$ & $-119.74(13)$ & $\mathrm{O} 1-\mathrm{N} 1-\mathrm{C} 4-\mathrm{C} 9$ & $-61.12(18)$ \\
\hline $\mathrm{O} 1-\mathrm{N} 1-\mathrm{C} 1-\mathrm{C} 8$ & $-61.57(17)$ & $\mathrm{C} 1-\mathrm{N} 1-\mathrm{C} 4-\mathrm{C} 9$ & $119.77(13)$ \\
\hline $\mathrm{C} 4-\mathrm{N} 1-\mathrm{C} 1-\mathrm{C} 8$ & $117.54(13)$ & $\mathrm{C} 2-\mathrm{C} 3-\mathrm{C} 4-\mathrm{N} 1$ & $-1.34(17)$ \\
\hline $\mathrm{N} 1-\mathrm{C} 1-\mathrm{C} 2-\mathrm{C} 3$ & $0.11(17)$ & $\mathrm{C} 5-\mathrm{C} 3-\mathrm{C} 4-\mathrm{N} 1$ & $178.28(14)$ \\
\hline $\mathrm{C} 7-\mathrm{C} 1-\mathrm{C} 2-\mathrm{C} 3$ & $117.23(15)$ & $\mathrm{C} 2-\mathrm{C} 3-\mathrm{C} 4-\mathrm{C} 10$ & $114.75(15)$ \\
\hline $\mathrm{C} 8-\mathrm{C} 1-\mathrm{C} 2-\mathrm{C} 3$ & $-116.35(15)$ & $\mathrm{C} 5-\mathrm{C} 3-\mathrm{C} 4-\mathrm{C} 10$ & $-65.63(18)$ \\
\hline $\mathrm{C} 1-\mathrm{C} 2-\mathrm{C} 3-\mathrm{C} 5$ & $-178.76(15)$ & $\mathrm{C} 2-\mathrm{C} 3-\mathrm{C} 4-\mathrm{C} 9$ & $-118.07(15)$ \\
\hline $\mathrm{C} 1-\mathrm{C} 2-\mathrm{C} 3-\mathrm{C} 4$ & $0.82(19)$ & $\mathrm{C} 5-\mathrm{C} 3-\mathrm{C} 4-\mathrm{C} 9$ & $61.54(18)$ \\
\hline $\mathrm{O} 1-\mathrm{N} 1-\mathrm{C} 4-\mathrm{C} 3$ & $-179.43(13)$ & & \\
\hline
\end{tabular}




\section{supporting information}

Hydrogen-bond geometry $\left(A,{ }^{\circ}\right)$

\begin{tabular}{lllll}
\hline$D-\mathrm{H} \cdots A$ & $D-\mathrm{H}$ & $\mathrm{H} \cdots A$ & $D \cdots A$ & $D-\mathrm{H} \cdots A$ \\
\hline $\mathrm{C} 2-\mathrm{H} 2 A \cdots \mathrm{O} 1^{\mathrm{i}}$ & $0.975(19)$ & $2.441(18)$ & $3.3907(18)$ & $164.6(14)$ \\
$\mathrm{C} 6-\mathrm{H} 6 A \cdots \mathrm{O} 1^{\mathrm{ii}}$ & $0.98(2)$ & $2.20(2)$ & $3.174(2)$ & $171.2(17)$ \\
\hline
\end{tabular}

Symmetry codes: (i) $x, y, z-1$; (ii) $x-1, y, z-1$. 